\title{
Clinical features and outcomes of patients with lung cancer as well as combined pulmonary fibrosis and emphysema
}

\author{
HAJIME OTSUKA $^{1}$, KEISHI SUGINO ${ }^{2}$, YOSHINOBU HATA ${ }^{1}$, TAKASHI MAKINO ${ }^{1}$, SATOSHI KOEZUKA ${ }^{1}$, \\ KAZUTOSHI ISOBE ${ }^{2}$, NAOBUMI TOCHIGI ${ }^{3}$, KAZUTOSHI SHIBUYA $^{3}$, SAKAE HOMMA $^{2}$ and AKIRA IYODA ${ }^{1}$ \\ Divisions of ${ }^{1}$ Chest Surgery and ${ }^{2}$ Respiratory Medicine; ${ }^{3}$ Department of Surgical Pathology, \\ Toho University School of Medicine, Tokyo 143-8541, Japan
}

Received March 28, 2016; Accepted May 20, 2016

DOI: $10.3892 / \operatorname{mco} .2016 .954$

\begin{abstract}
The syndrome of combined pulmonary fibrosis and emphysema (CPFE) has been characterized by severely impaired gas exchange and poor survival. However, the clinical features of patients with lung cancer plus CPFE have remained elusive. The present study performed a retrospective analysis to examine the clinical characteristics and outcome of surgically resected patients with lung cancer plus CPFE. Among 831 patients with primary lung cancer who underwent surgical resection, 23 patients $(2.8 \%)$ were diagnosed with CPFE and 9 patients (1.1\%) with solely idiopathic pulmonary fibrosis (IPF). Thirty-five patients were stratified as the solely emphysema group with adjustment of the pathological stage. The clinicopathological characteristics of patients in the CPFE group and their outcomes were evaluated and compared with those with the solely IPF or solely emphysema groups. Within the CPFE group, no significant differences in survival between patients with post-operative acute exacerbation (AE; $\mathrm{n}=3)$ and those without $\mathrm{AE}(\mathrm{n}=20)$ were noted; however, in the solely IPF group, patients with post-operative AE $(n=4)$ had a significantly shorter survival than those without $\mathrm{AE}(\mathrm{n}=5$; $\mathrm{P}=0.022$ ). The 5-year survival rate of patients in the CPFE, solely IPF and solely emphysema groups was 22, 22 and 58\%, respectively. Furthermore, the CPFE and solely IPF groups showed a significantly shorter survival than the solely emphysema group $(\mathrm{P}=0.001$ and 0.011 , respectively). In conclusion, surgically resected lung cancer patients with CPFE had poor survival, which was, in contrast to that of lung cancer patients with solely IPF, not affected by AE.
\end{abstract}

Correspondence to: Professor Akira Iyoda, Division of Chest Surgery, Toho University School of Medicine, 6-11-1 Omori-nishi, Ota-ku, Tokyo 143-8541, Japan

E-mail: aiyoda@med.toho-u.ac.jp

Key words: emphysema, lung cancer, surgery, interstitial lung disease, combined pulmonary fibrosis and emphysema

\section{Introduction}

The syndrome of combined pulmonary fibrosis and emphysema (CPFE) has been suggested to be a clinically important phenotype of pulmonary fibrosis (1). Cottin et al (1) defined CPFE as a distinct condition, with characteristic features in the chest computed tomography (CT) image that include emphysema in the upper pulmonary region concomitant with parenchymal fibrosis in the lower region. The syndrome is characterized by sub-normal spirometry, severely impaired gas exchange, high prevalence of pulmonary hypertension and poor survival (2-4). Mejía et al (3) reported that survival of CPFE patients was poorer compared with that of patients with idiopathic pulmonary fibrosis (IPF) alone and that pulmonary hypertension in CPFE patients was an independent predictor of mortality. Previous studies have indicated that CPFE patients have a significantly increased risk of lung cancer compared with patients with chronic obstructive pulmonary disease (COPD) or IPF alone $(1,4,5)$. Post-operative acute exacerbation (AE) of interstitial lung disease in patients with lung cancer is associated with a high mortality rate (43.9\%) (6). However, the clinical features and prognosis of patients with lung cancer and CPFE have remained elusive. The present study performed a retrospective analysis to determine the prevalence, clinical characteristics and outcome of patients with surgically resected lung cancer and CPFE. In addition, differences between the clinical features and outcomes of patients with lung cancer and CPFE, solely IPF and solely emphysema were assessed, and the impact of post-operative AE on patient survival was determined.

\section{Patients and methods}

Patient data. Of 831 consecutive patients with primary lung cancer who underwent surgical resection at Toho University School of Medicine between June 2004 to December 2014, 23 patients with CPFE (2.8\%) were retrospectively reviewed by the present study. CPFE was diagnosed by chest high-resolution computed tomography (HRCT) images showing emphysema involving $>25 \%$ of both upper lobes and a usual interstitial pneumonia (UIP) pattern of fibrosis in the lower lobes (Fig. 1). Histological examination of resected specimens and/or diagnosis of IPF based on a high-resolution CT scan 
showing honeycombing (multiple equal-sized cystic lesions of 2-10 $\mathrm{mm}$ with a thick wall) in the sub-pleural area of both lung fields according to the American Thoracic Society/European Respiratory Society International Consensus Statement on IPF were used to confirm the presumptive diagnosis based on the UIP pattern (7). AE was diagnosed according to the guidelines of the Japanese Respiratory Society (8) based on the following criteria fulfilled over one month: i) Increased respiratory distress; ii) fibrosis, newly developed ground glass opacity and an infiltrative shadow in the HRCT scan; and iii) a decrease in arterial oxygen pressure of more than 10 Torr under constant oxygenation conditions. Acute deterioration triggered by anti-cancer drug (carboplatin/etoposide, carboplatin/paclitaxel/bevacizumab, gefitinib) and AE as natural courses of IPF (16 months after the surgery) were excluded from post-operative AE. The cumulative amount of cigarette consumption was expressed as pack years, calculated as the number of cigarette packs consumed per day multiplied by the number of years of smoking. For analysis, patients in the CPFE group were further stratified into a group which developed post-operative $\mathrm{AE}(\mathrm{n}=3)$ and another group without $\mathrm{AE}$ $(n=20)$. For comparison, patients with IPF other than CPFE were selected from the abovementioned cohort as the solely IPF group (n=9), which was further divided into an IPF with post-operative $\mathrm{AE}$ group $(\mathrm{n}=4)$ and one group without $\mathrm{AE}$ $(n=5)$. Furthermore, 35 patients with COPD and emphysema observed by chest HRCT were defined as the solely emphysema group with adjustment of the pathological stage. The present study was approved by the local Ethics committee of Toho University Omori Medical Center (assurance no. 26-232).

Measurement of serum marker levels. As indicators of UIP, the serum levels of Krebs von den Lungen-6 (KL-6) and surfactant protein-D (SP-D) were determined prior to surgery. Serum KL-6 (normal levels, $<500 \mathrm{U} / \mathrm{ml}$ ) was measured using an enzyme-linked immunosorbent assay (ELISA; ELTEST KL-6 kit; Eisai Co., Tokyo, Japan) and serum SP-D (normal levels, $<110 \mathrm{ng} / \mathrm{ml}$ ) was measured using an ELISA kit (SP-D kit YAMASA EIA II, Yamasa Corp., Tokyo, Japan).

Chest CT. Chest CT was performed using a helical CT scanner (Aquilion 16; Toshiba, Tokyo, Japan). Routine scanning of the entire lung was performed with a slice thicknesses of 5-10 mm, followed by HRCT imaging at full inspiration with 1-2-mm sections (120 kVp; $300 \mathrm{~mA}$; pitch, 1.0). For all patients, HRCT images were captured with a window setting appropriate for the lungs (window level from -600 Hounsfield units; width from 1,600 Hounsfield units).

Pulmonary function testing. Spirometry and measurement of the diffusing capacity of the lung for carbon monoxide (DLco) were performed using a pulmonary function test system (Chestac-33; CHEST Co. Ltd., Tokyo, Japan). The DLco was measured using the single-breath technique. The pulmonary function tests were performed by two technicians according to the recommendations of the American Thoracic Society (9).

Statistical analysis. All statistical analyses were performed using the JMP version 10.0.0 statistical software package
(SAS Institute, Cary, NC, USA). Comparison of categorical and dichotomous variables was performed using Pearson's $\chi^{2}$ test or Fisher's exact test. Analysis of variance with life tables and Kaplan-Meier curves were used for the analyses of overall survival. Differences between two groups were analyzed using the log-rank test. $\mathrm{P}<0.05$ was considered to indicate a statistically significant difference.

\section{Results}

Patient characteristics. The characteristics of the patients are shown in Table I. All patients in the CPFE or solely IPF groups were male, while five patients in the emphysema group were female (14\%). The results of the pulmonary function tests, including the percent vital capacity (\%VC) or forced expiratory volume in one second as percent of forced vital capacity $\left(\mathrm{FEV}_{1.0} \%\right)$ of the patients in the CPFE group were almost normal, while patients in the solely IPF group had a slightly decreased \% VC and patients in the solely emphysema had a decreased $\mathrm{FEV}_{1.0} \%$ and $\% \mathrm{FEV}_{1.0}$. However, the percent predicted DLco (\%DLco) of the patients in the CPFE group was noticeably low. The predominant histology of lung tumors in the CPFE group was squamous-cell carcinoma and the majority of patients had advanced-stage disease. No statistically significant differences were observed in the mean age, smoking status, \%VC, $\mathrm{FEV}_{1.0} \%$, \%DLco, type of surgical procedure, histological type and pathological stage of lung cancer between patients in the CPFE with AE group and those in the CPFE without AE group (Table II).

Survival. Survival in the CPFE and solely IPF groups is significantly lower compared with that in the solely emphysema group, while AE does not affect survival in the CPFE group. The 3- and 5-year survival rate in the CPFE group was 38 and $22 \%$, respectively. The Kaplan-Meier survival curves for patients in the CPFE group showed no significant differences between patients with $\mathrm{AE}$ and those without $\mathrm{AE}$ $(\mathrm{P}=0.084$; Fig. 2A).The 3-year survival rate in the CPFE with $\mathrm{AE}$ group was $33 \%$ and that in the CPFE without AE group was $38 \%$. The survival time in the solely IPF with AE group was significantly shorter compared with that in the solely IPF without AE group (5.0 vs. 24.4 month $\mathrm{P}=0.022$, log-rank test; Fig. 2B). The 5-year survival rate of patients in the CPFE, solely IPF and solely emphysema groups was 22, 22 and $58 \%$, respectively. Furthermore, the CPFE and solely IPF groups showed a significantly shorter survival than the solely emphysema group ( $\mathrm{P}=0.001$ and 0.011 , respectively; Fig. 3); however, no significant difference was determined between the CPFE and solely IPF group $(P=0.924$. In Tables III and IV, data on the fatal cases in the CPFE with AE and CPFE without AE groups are summarized, respectively. In the CPFE with AE group, two patients succumbed to post-operative AE after 2 months and one patient succumbed after 36 months (Table III). In the CPFE without AE group, four patients succumbed to lung cancer and two succumbed to other cancer types. Furthermore, the cause of mortality was chemotherapy-induced AE in three patients (carboplatin/ etoposide, carboplatin/paclitaxel/bevacizumab, gefitinib), pneumonia in another three patients and myocardial infarction in one patient (Table IV). 
Table I. Patient characteristics.

\begin{tabular}{|c|c|c|c|c|c|}
\hline Characteristic & $\begin{array}{l}\text { CPFE } \\
(\mathrm{n}=23)\end{array}$ & $\begin{array}{l}\text { Solely IPF } \\
\qquad(\mathrm{n}=9)\end{array}$ & $\begin{array}{l}\text { Solely Em } \\
\quad(\mathrm{n}=35)\end{array}$ & $\begin{array}{c}\text { P-value } \\
\text { (CPFE vs. IPF) }\end{array}$ & $\begin{array}{c}\text { P-value } \\
\text { (CPFE vs. Em) }\end{array}$ \\
\hline Age (years), mean \pm SD & $69.4 \pm 6.7$ & $72.1 \pm 6.8$ & $70.3 \pm 6.7$ & 0.323 & 0.649 \\
\hline Male/female & $23 / 0$ & 9/0 & $30 / 5$ & - & 0.146 \\
\hline \multicolumn{6}{|l|}{ Smoking status } \\
\hline Current/former/never & $6 / 17 / 0$ & $3 / 6 / 0$ & $13 / 20 / 2$ & 0.685 & 0.206 \\
\hline Pack years & $50 \pm 27$ & $37 \pm 20$ & $53 \pm 23$ & 0.201 & 0.707 \\
\hline \multicolumn{6}{|l|}{ Pulmonary function } \\
\hline$\% \mathrm{VC}(\%)$ & $102 \pm 19$ & $89 \pm 21$ & $98 \pm 18$ & 0.114 & 0.409 \\
\hline $\mathrm{FEV}_{1.0} \%(\%)$ & $72 \pm 10$ & $82 \pm 8$ & $59 \pm 9$ & 0.009 & $<0.001$ \\
\hline$\% \mathrm{FEV}_{1.0}(\%)$ & $103 \pm 21$ & $103 \pm 27$ & $80 \pm 21$ & 0.992 & $<0.001$ \\
\hline$\%$ DLco $(\%)$ & $68 \pm 19$ & $73 \pm 16$ & $83 \pm 20$ & 0.494 & 0.009 \\
\hline KL-6 (U/ml) & $673 \pm 393$ & $821 \pm 241$ & - & 0.309 & - \\
\hline SP-D (ng/ml) & $135 \pm 90$ & $165 \pm 73$ & - & 0.397 & - \\
\hline \multicolumn{6}{|l|}{ Surgical procedure } \\
\hline Lobectomy/limited & $20 / 3$ & $8 / 1$ & $33 / 2$ & 1.000 & 0.376 \\
\hline \multicolumn{6}{|l|}{ Histology } \\
\hline $\mathrm{Sq} / \mathrm{Ad} /$ other & $13 / 5 / 5$ & $3 / 4 / 2$ & $20 / 13 / 2$ & 0.398 & 0.135 \\
\hline \multicolumn{6}{|l|}{ Pathological stage } \\
\hline $\mathrm{I} / \mathrm{II} / \mathrm{III}$ & $6 / 10 / 7$ & $2 / 4 / 3$ & $9 / 15 / 11$ & 0.971 & 0.997 \\
\hline
\end{tabular}

P-values were calculated using Student $t$-test or Chi-square test. Em, emphysema; IPF, idiopathic pulmonary fibrosis; CPFE, combined pulmonary fibrosis and emphysema; $\mathrm{SD}$, standard deviation; \%VC, percent vital capacity; $\mathrm{FEV}_{1.0} \%$, forced expiratory volume in one second as percent of forced vital capacity; $\% \mathrm{FEV}_{1.0}$, percent predicted forced expiratory volume in one second; \%DLco, percent predicted diffusing capacity of the lung for carbon monoxide; KL-6, Krebs von den Lungen-6; SP-D, surfactant protein-D; Sq, squamous-cell carcinoma; Ad, adenocarcinoma.

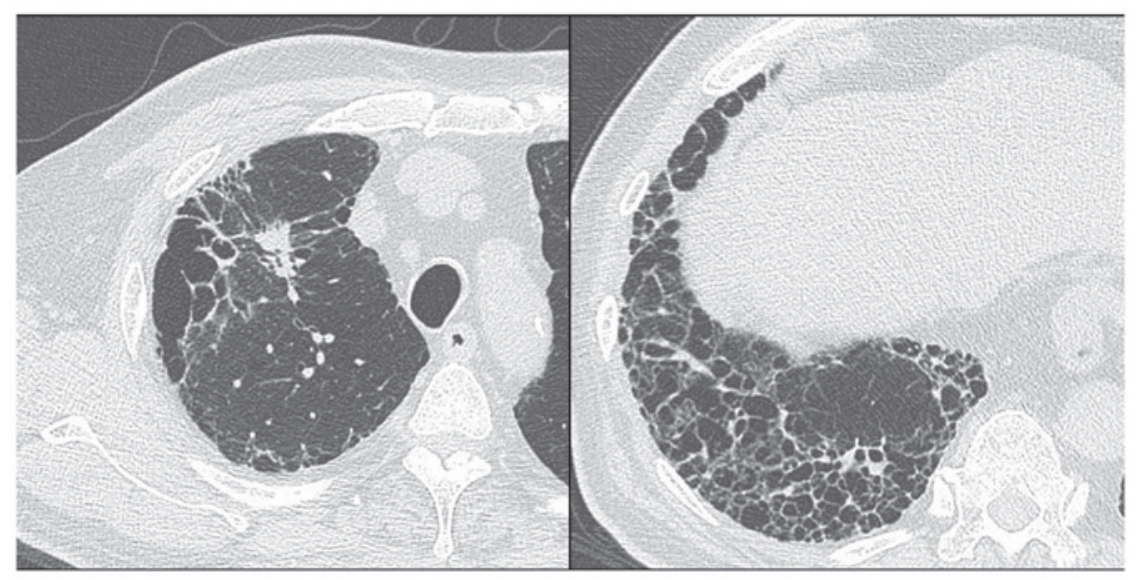

Figure 1. High-resolution computed tomography scan of a patient with combined pulmonary fibrosis and emphysema, who presented with an enlarged nodule in the right upper lobe (left). The image shows emphysema involving $>25 \%$ of both upper lobes and a usual interstitial pneumonia pattern of fibrosis in the lower lobes (right).

\section{Discussion}

As first defined by Cottin et al (1) in 2005, CPFE is a well-defined syndrome characterized by upper-lobe-predominant emphysema and lower-lobe-predominant fibrosis observed by chest HRCT. The median survival of CPFE patients has been reported to range from 22 months to 8 years and the 5 -year survival rate is $22-80 \%(1,4,10-12)$. Mejía et al (3) reported that $31 / 110$ patients with IPF (28.2\%) had emphysema and that their mortality rate was higher compared with that of patients with IPF alone.

Previous studies have shown that the risk of primary lung cancer is significantly increased in patients with CPFE. Kitaguchi et al (2) reported that in a cohort with CPFE, 
A

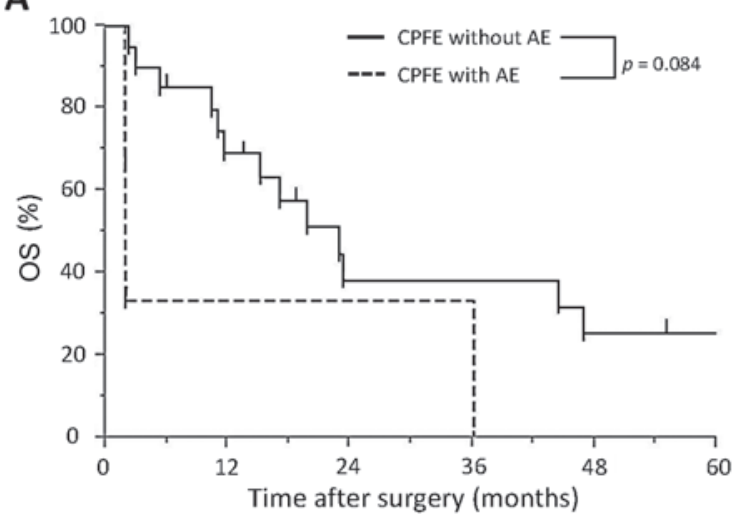

B

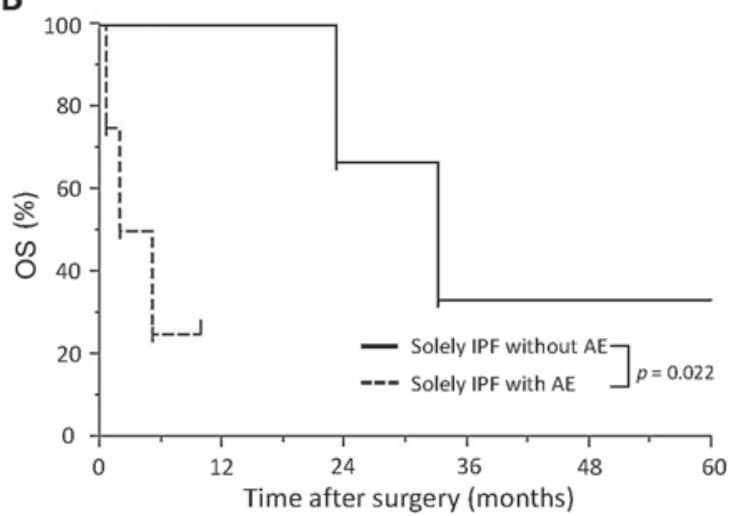

Figure 2. OS in various patient groups. (A) Kaplan-Meier estimates of overall survival of lung cancer patients with combined pulmonary fibrosis and emphysema divided into those who developed post-operative $\mathrm{AE}(\mathrm{n}=3)$ and those who did not $(\mathrm{n}=20)$. The 3 -year survival rates were $33 \%$ in patients with $\mathrm{AE}$ and $38 \%$ in those without AE, while differences were not significant ( $\mathrm{P}=0.084, \log$-rank test). (B) Kaplan-Meier estimates of overall survival of lung cancer patients with solely IPF divided into those who developed AE $(n=4)$ and those who did not $(n=5)$. The survival time of patients with IPF and AE was significantly shorter compared with that of patients without $\mathrm{AE}(\mathrm{P}=0.022$, log-rank test). OS, overall survival; AE, acute exacerbation; IPF, idiopathic pulmonary fibrosis.

Table II. Characteristics of CPFE with $\mathrm{AE}$ and without $\mathrm{AE}$ groups.

\begin{tabular}{|c|c|c|c|}
\hline Characteristic & $\begin{array}{c}\mathrm{AE} \\
(\mathrm{n}=3)\end{array}$ & $\begin{array}{l}\text { Without AE } \\
\quad(n=20)\end{array}$ & P-value \\
\hline Age (years), mean \pm SD & $66.7 \pm 8.6$ & $69.9 \pm 6.6$ & 0.459 \\
\hline Male/female & $3 / 0$ & $20 / 0$ & - \\
\hline \multicolumn{4}{|l|}{ Smoking status } \\
\hline Former/current & $3 / 0$ & $14 / 6$ & 0.539 \\
\hline Pack years & $44 \pm 17$ & $51 \pm 28$ & 0.687 \\
\hline \multicolumn{4}{|l|}{ Pulmonary function } \\
\hline$\% \mathrm{VC}(\%)$ & $95 \pm 10$ & $103 \pm 20$ & 0.504 \\
\hline$\% \mathrm{FEV}_{1.0}(\%)$ & $96 \pm 12$ & $104 \pm 22$ & 0.558 \\
\hline $\mathrm{FEV}_{1.0} \%(\%)$ & $77 \pm 13$ & $71 \pm 10$ & 0.345 \\
\hline$\% \operatorname{DLco}(\%)$ & $65 \pm 3$ & $69 \pm 21$ & 0.761 \\
\hline KL-6 (U/ml) & $486 \pm 149$ & $706 \pm 416$ & 0.386 \\
\hline SP-D (ng/ml) & $134 \pm 55$ & $135 \pm 96$ & 0.984 \\
\hline \multicolumn{4}{|l|}{ Surgical procedure } \\
\hline Lobectomy/limited & $2 / 1$ & $18 / 2$ & 0.320 \\
\hline \multicolumn{4}{|l|}{ Histology } \\
\hline $\mathrm{Sq} / \mathrm{Ad} /$ other & $2 / 0 / 1$ & $11 / 5 / 4$ & 0.439 \\
\hline \multicolumn{4}{|l|}{ Pathological stage } \\
\hline I/II/III & $2 / 0 / 1$ & $4 / 10 / 6$ & 0.109 \\
\hline
\end{tabular}

P-values were calculated using Student $t$-test or Chi-square test. AE, acute exacerbation; CPFE, combined pulmonary fibrosis and emphysema; SD, standard deviation; \%VC, percent vital capacity; $\mathrm{FEV}_{1.0} \%$, forced expiratory volume in one second as percent of forced vital capacity; $\% \mathrm{FEV}_{1.0}$, percent predicted forced expiratory volume in one second; \%DLco, percent predicted diffusing capacity of the lung for carbon monoxide; KL-6, Krebs von den Lungen-6; SP-D, surfactant protein-D; Sq, squamous-cell carcinoma; Ad, adenocarcinoma.

the prevalence of lung cancer was significantly increased compared with that in a cohort with COPD alone (46.8 vs. 7.3\%; $\mathrm{P}<0.01$ ), and Kurashima et al (11) identified a significantly

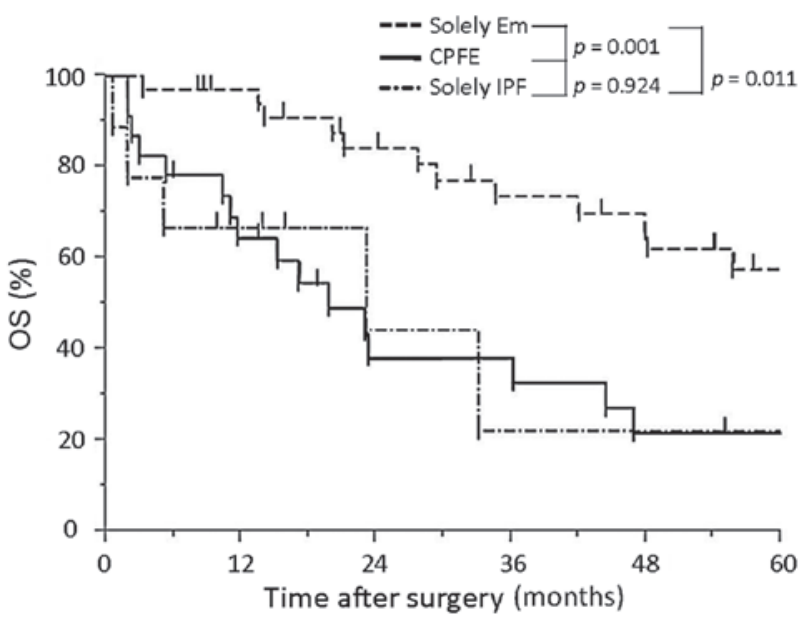

Figure 3. OS of patients with CPFE, solely IPF and solely Em. The 5-year survival rates were 22,22 and $58 \%$, respectively. Patients with CPFE or solely IPF showed a significantly poor survival compared with those with solely $\operatorname{Em}(\mathrm{P}=0.001$ and 0.011 , respectively). OS, overall survival; Em, emphysema; IPF, idiopathic pulmonary fibrosis; CPFE, combined pulmonary fibrosis and emphysema.

increased rate of lung cancer-associated mortality among patients with CPFE (12 of 36 mortalities, 33.3\%) compared with that in patients with IPF alone (8 of 66 mortalities, $12.1 \%$; $\mathrm{P}=0.0097)$. Sugino et al (4) suggested that the following factors or diagnostic errors may account for the differential outcomes of CPFE and IPF alone: i) The presence of primary lung cancer, ii) disease severity based on distributions or extension of fibrosis and/or emphysema, iii) possible confusion between chronic fibrotic interstitial pneumonia and fibrotic non-specific interstitial pneumonia, iv) the difficulty in radiological discrimination between emphysema with non-specific fibrosis and IPF (for example, wall thickening due to emphysematous may be mistaken for honeycomb cysts). The study also reported that patients with CPFE had a poor outcome even in patients without the complication of lung cancer at the initial visit, with median survival time of 28 months in their subgroup analysis (4). However, these findings suggested 
Table III. Fatal cases in the combined pulmonary fibrosis and emphysema with post-operative acute exacerbation group.

\begin{tabular}{llllccrc}
\hline $\begin{array}{l}\text { Age } \\
\text { (years) }\end{array}$ & $\begin{array}{c}\text { Surgical } \\
\text { procedure }\end{array}$ & Stage & Histology & Trigger of AE & $\begin{array}{c}\text { Time between } \\
\text { surgery and AE } \\
\text { (months) }\end{array}$ & $\begin{array}{c}\text { Survival } \\
\text { (months) }\end{array}$ & $\begin{array}{c}\text { Cause } \\
\text { of mortality }\end{array}$ \\
\hline 59 & Lobectomy & pIB & AdSq & Surgery & 0.3 & 36 & AE \\
65 & Limited & pIA & Sq & Surgery & 1.4 & 2 & AE \\
76 & Lobectomy & pIIIA & Sq & Surgery & 0.2 & 2 & AE \\
\hline
\end{tabular}

$\mathrm{AE}$, acute exacerbation; Sq, squamous cell-carcinoma; AdSq, adenosquamous carcinoma.

Table IV. Fatal cases in the combined pulmonary fibrosis and emphysema group without post-operative acute exacerbation.

\begin{tabular}{lllclcc}
\hline $\begin{array}{l}\text { Age } \\
\text { (years) }\end{array}$ & $\begin{array}{l}\text { Surgical } \\
\text { procedure }\end{array}$ & Stage & Histology & Recurrent site & $\begin{array}{c}\text { Survival } \\
\text { (months) }\end{array}$ & $\begin{array}{c}\text { Cause of } \\
\text { mortality }\end{array}$ \\
\hline 63 & Lobectomy & pIIIA & Sq & Lung & 29 & Lung cancer \\
65 & Lobectomy & pIIB & Sq & Mediastinum & 47 & $\begin{array}{l}\text { Lung cancer } \\
\text { Lung cancer }\end{array}$ \\
68 & Lobectomy & pIB & Sm & Lymph node & 23 & Lung cancer \\
81 & Lobectomy & pIIB & Ad & Mediastinum & 17 & AE \\
64 & Lobectomy & pIA & Sm & - & 11 & AE \\
65 & Lobectomy & pIB & Ad & - & 44 & AE \\
71 & Lobectomy & pIIA & Ad & Pleural dissemination & 2 & Pneumonia \\
61 & Lobectomy & pIIB & Sq & - & 12 & Pneumonia \\
61 & Lobectomy & pIIB & Sq & - & 10 & Pneumonia \\
69 & Lobectomy & pIIIA & Ad & - & 23 & Other cancer type \\
61 & Lobectomy & pIIIA & Sq & - & 16 & Other cancer type \\
73 & Limited & pIIIA & Sq & - & 4 & Myocardial infarction \\
75 & Limited & pIB & Sm & - & & 13 \\
\hline
\end{tabular}

AE, acute exacerbation (chemotherapy-induced); Sq, squamous cell-carcinoma; Ad, adenocarcinoma; Sm, small-cell carcinoma.

that CPFE is associated with a poor prognosis, which was in accordance with the present study, which reported poor 3- and 5 -year survival rates of 38 and $22 \%$, respectively for resected $\mathrm{CPFE}$ patients with lung cancer. Of note, a number of CPFE patients in the present study succumbed to AE or lung cancer recurrence. However, as shown in Table I, the pulmonary function of these patients was almost normal, except for the DLco results. CPFE may be difficult to detect by the pulmonary function tests that are usually performed for patients. DLco is important to detect CPFE and to consider the potential risk of chemotherapy for the patients with CPFE.

$\mathrm{AE}$ is an important negative prognostic factor for surgically resected lung cancer patients with interstitial lung disease (ILD). Sato et al (6) reported that AE occurred in $9.3 \%$ of surgically resected lung cancer patients with ILD, accounting for a mortality rate of $43.9 \%$. They also identified the following independent risk factors for AE: Surgical procedures (wedge resection, lobectomy/segmentectomy, bilobectomy/pneumonectomy), male gender, history of exacerbation, pre-operative steroid use, elevated serum KL-6 levels $(\geq 1,000 \mathrm{U} / \mathrm{ml})$, appearance of UIP in the CT spectrum and reduced percentage of predicted vital capacity. Although two of the patients in the present study matched the risk factors of male gender and CT findings, it was not possible to determine whether these or any other factors were associated with CPFE due to the low patient number. In the present study, only three cases with post-operative AE were included and no statistically significant differences in clinicopathological parameters or survival rates between patients with $\mathrm{AE}$ and those without AE were identified. Previous studies have reported that surgically resected lung cancer patients with IPF who developed post-operative AE had a poorer survival compared with those without AE (13-16), which was in line with the findings of the present study regarding the solely IPF with AE and the solely IPF without AE groups. The finding that AE did not affect survival in CPFE patients in the present study may be due to inclusion of cases of small-cell carcinoma in the CPFE without AE group, in addition to the small sample size. Therefore, additional analysis is required, including the comparison of the molecular characteristics of lung cancer patients with CPFE and those with lung cancer and IPF.

The present study had several limitations. There were intrinsic limitations associated with the data, as they were collected and reviewed retrospectively. In addition, the study cohort was small, and further studies on larger populations are required to validate the results. 
In conclusion, the present study revealed that lung cancer patients with CPFE had poor survival, which was comparable with that of lung cancer patients with IPF. Numerous patients with lung cancer and CPFE presented with post-operative recurrence of lung cancer or developed AE or pneumonia. The present study contributed to the current understanding of the clinical and pathophysiological features as well as the outcome of lung cancer patients with CPFE, which requires to be confirmed and further elucidated by larger-scale studies.

\section{Acknowledgements}

The present study was supported in part by Grants-in-aid for Scientific Research [nos. (C) 15K10272 and 26462140] from the Japanese Ministry of Education, Culture, Sports, Science and Technology, as well as by a Grant-in-aid for Project Research from Toho University School of Medicine (no. 26-14) and the Research Promotion Grant from Toho University Graduate School of Medicine (no. 15-02).

\section{References}

1. Cottin V, Nunes H, Brillet PY, Delaval P, Devouassoux G, Tillie-Leblond I, Israel-Biet D, Court-Fortune I, Valeyre D and Cordier JF; Groupe d'Etude et de Recherche sur les Maladies Orphelines Pulmonaires (GERM O P): Combined pulmonary fibrosis and emphysema: A distinct underrecognised entity. Eur Respir J 26: 586-593, 2005.

2. Kitaguchi Y, Fujimoto K, Hanaoka M, Kawakami S, Honda T and Kubo K: Clinical characteristics of combined pulmonary fibrosis and emphysema. Respirology 15: 265-271, 2010.

3. Mejía M, Carrillo G, Rojas-Serrano J, Estrada A, Suárez T, Alonso D, Barrientos E, Gaxiola M, Navarro C and Selman M: Idiopathic pulmonary fibrosis and emphysema: Decreased survival associated with severe pulmonary arterial hypertension. Chest 136: 10-15, 2009.

4. Sugino K, Ishida F, Kikuchi N, Hirota N, Sano G, Sato K, Isobe K, Sakamoto S, Takai Y and Homma S: Comparison of clinical characteristics and prognostic factors of combined pulmonary fibrosis and emphysema versus idiopathic pulmonary fibrosis alone. Respirology 19: 239-245, 2014.

5. Dai H, Liu J, Liang L, Ban C, Jiang J, Liu Y, Ye Q and Wang C: Lung cancer risk among patients with combined pulmonary fibrosis and emphysema. Respirology 19: 707-713, 2014.
6. Sato T, Teramukai S, Kondo H, Watanabe A, Ebina M, Kishi K, Fujii Y, Mitsudomi T, Yoshimura M, Maniwa T, et al: Impact and predictors of acute exacerbation of interstitial lung diseases after pulmonary resection for lung cance. $J$ Thorac Cardiovasc Surg 147: 1604-1611, 2014.

7. Raghu G, Collard HR, Egan JJ, Martinez FJ, Behr J, Brown KK, Colby TV, Cordier JF, Flaherty KR, Lasky JA, et al: An official ATS/ERS/JRS/ALAT statement: Idiopathic pulmonary fibrosis: Evidence-based guidelines for diagnosis and management. Am J Respir Crit Care Med 183: 788-824, 2011.

8. Akira M, Hamada H, Sakatani M, Kobayashi C, Nishioka M and Yamamoto S: CT findings during phase of accelerated deterioration in patients with idiopathic pulmonary fibrosis. AJR Am J Roentgenol 168: 79-83, 1997.

9. Single breath carbon monoxide diffusing capacity (transfer factor): Recommendations for a standard technique. Statement of the American thoracic society. Am Rev Respir Dis 136: 1299-1307, 1987.

10. Akagi T, Matsumoto T, Harada T, Tanaka M, Kuraki T, Fujita $\mathrm{M}$ and Watanabe $\mathrm{K}$ : Coexistent emphysema delays the decrease of vital capacity in idiopathic pulmonary fibrosis. Respir Med 103: 1209-1215, 2009.

11. Kurashima K, Takayanagi N, Tsuchiya N, Kanauchi T, Ueda M, Hoshi T, Miyahara Y and Sugita Y: The effect of emphysema on lung function and survival in patients with idiopathic pulmonary fibrosis. Respirology 15: 843-848, 2010.

12. Ryerson CJ, Hartman T, Elicker BM, Ley B, Lee JS, Abbritti M, Jones KD, King TE Jr and Ryu J, Collard HR: Clinical features and outcomes in combined pulmonary fibrosis and emphysema in idiopathic pulmonary fibrosis. Chest 144: 234-240, 2013.

13. Chiyo M, Sekine Y, Iwata T, Tatsumi K, Yasufuku K, Iyoda A, Otsuji M, Yoshida S, Shibuya K, Iizasa T, et al: Impact of interstitial lung disease on surgical morbidity and mortality for lung cancer: Analyses of short-term and long-term outcomes. J Thorac Cardiovasc Surg 126: 1141-1146, 2003.

14. Kumar P, Goldstraw P, Yamada K, Nicholson AG, Wells AU, Hansell DM, Dubois RM and Ladas G: Pulmonary fibrosis and lung cancer: Risk and benefit analysis of pulmonary resection. J Thorac Cardiovasc Surg 125: 1321-1327, 2003.

15. Koizumi K, Hirata T, Hirai K, Mikami I, Okada D, Yamagishi S, Kawashima T, Kinoshita H, Enomoto Y, Nakajima Y and Shimizu K: Surgical treatment of lung cancer combined with interstitial pneumonia: The effect of surgical approach on postoperative acute exacerbation. Ann Thorac Cardiovasc Surg 10: 340-346, 2004

16. Watanabe A, Kawaharada $\mathrm{N}$ and Higami T: Postoperative acute exacerbation of IPF after lung resection for primary lung cancer. Pulm Med 2011: 960316, 2011. 